\title{
Large Deviations for Multiplicative Chaos
}

\section{P. Collet and F. Koukiou *}

Centre de Physique Théorique ${ }^{\star}$, Ecole Polytechnique, F-91128 Palaiseau Cedex, France

Received February 25, 1991; in revised form December 12, 1991

\begin{abstract}
The local singularities for a class of random measures, obtained by random iterated multiplications, are investigated using the thermodynamic formalism. This analysis can be interpreted as a rigorous study of the phase transition of a system with random interactions.
\end{abstract}

\section{Introduction}

The notion of singularity of a measure was introduced in [6] in connection with potential theory. A typical singularity can be defined when it is associated to the capacity of the measure. It was recently realized that this analysis can be further developed for the invariant measure of some dynamical systems [2]. In this special situation, one can use expansive (respectively contractive) properties of the mapping to compare the singularities of a measure at different scales. Using the thermodynamic formalism, it was possible to obtain non-trivial results on the local singularities of a measure. This information is usually recorded in the so-called $f(\alpha)$ functions which can be defined as follows. Let $\mu$ be a Borel measure on $\mathbf{R}^{d}$. For any point $M$ in $\mathbf{R}^{d}$ we define two numbers $\alpha_{+}$and $\alpha_{-}$by

$$
\alpha_{+}(M)=\limsup _{\varepsilon \rightarrow 0} \frac{\log \left|\mu\left(B_{\varepsilon}(M)\right)\right|}{d \log \varepsilon} \quad \text { and } \quad \alpha_{-}(M)=\liminf _{\varepsilon \rightarrow 0} \frac{\log \left|\mu\left(B_{\varepsilon}(M)\right)\right|}{d \log \varepsilon},
$$

where $B_{\varepsilon}(M)$ is the ball centered at $M$ of radius $\varepsilon$. The numbers $\alpha_{+}(M)$ and $\alpha_{-}(M)$ describe the local singularity of the measure $\mu$ at $M$. For simple ergodic dynamical systems the two functions $\alpha_{+}$and $\alpha_{-}$are equal and constant $\mu$-almost surely (see [12] for a more general situation). It was however realised in [8] that there is some interesting information in the sets where $\alpha_{+}$and $\alpha_{-}$take a value $\alpha$ different from the typical one. If one defines the level sets $B_{\alpha}^{ \pm}$by

$$
B_{\alpha}^{ \pm}=\left\{M \mid \alpha_{ \pm}(M)=\alpha\right\},
$$

* Partially supported by SCIENCE grant CT000307

$\star \star$ UPR A014 du CNRS 
one gets a family of complicated sets, and it is natural to ask for the Hausdorff dimension of these sets. The values of these Hausdorff dimensions considered as functions of $\alpha$ give the so called $f(\alpha)$ curves when the sets $B_{\alpha}^{ \pm}$have the same Hausdorff dimensions. Under suitable hypothesis, this function $f(\alpha)$ is the Legendre transform of a pressure function $P(\beta)$ which is the main object studied in the present paper.

More recently, in the context of measure valued processes, the singularities of (random) measures are extensively studied as a function of time [17].

We shall consider in the present paper the problem of the local singularity for a class of random measures obtained as limits of martingales. This situation emerged in a discontinuous model for turbulence introduced by Mandelbrot [15] in terms of random iterated multiplications - known as Multiplicative Chaos - and analysed in detail by Kahane and Peyrière [10], and Guivarc'h [7] (see also [9] and [4] for recent results).

This particular class of measures is interesting because it raises some questions similar to those arising in statistical mechanics of spin systems with random interactions. The main analogy is connected with the thermodynamic formalism used to analyse the function $f_{ \pm}(\alpha)$. Although it is not claimed that the model we consider provides a faithful mathematical formalism for the spin glass problem it enlightens some of the difficulties encountered there. For some recent rigorous results in this direction see also $[1,13]$.

In Sect. 2 we introduce the model of Multiplicative Chaos as a problem of statistical mechanics. As we shall see this model undergoes a phase transition. We analyse the high temperature phase in Sect. 3. We show the existence and the self-averaging property of the free energy and we characterise the unique random state. The low temperature region is studied in Sect. 4. In particular, we derive an explicit formula for the free energy. We intend to study the structure of the low temperature state(s) in our next paper.

\section{The Model}

We now define the model to be studied $[7,10,15]$. Let $c$ be an integer bigger than 1 and $W$ a real bounded random variable defined on the probability space $(\Omega, \mathscr{F}, P)$, with mean 1 and such that $1 / W$ is a.s. bounded (we exclude of course the case where $W$ is a constant almost surely). Let $\mathscr{D}_{p}$ be the countable $c$-adic partition of the interval $[0,1]$ of order $p$, i.e.

$$
\mathscr{D}_{p}=\left\{\left[k c^{-p},(k+1) c^{-p}\left[, 0 \leqq k \leqq c^{p}-1\right\} .\right.\right.
$$

To each element $I$ of $\mathscr{D}=\bigcup_{p=1}^{\infty} \mathscr{D}_{p}$, we associate the random variable $W_{I}$ having common distribution with $W$. We shall assume that if $I, J \in \mathscr{D}, I \cap J=\emptyset, W_{I}$ and $W_{J}$ are independent. On the $\sigma$-field generated by $\mathscr{D}_{n}$, we define a sequence of random measures $\mu_{n}$ at the "inverse temperature" $\beta$ as follows. If $I$ is an atom of $\mathscr{D}_{n}$, we set

$$
\mu_{\beta, n}(I)=c^{-n \beta} \prod_{s=1}^{n} \sum_{J \in \mathscr{Q}_{s}} W_{J}^{\beta} \lambda(J \cap I) c^{n},
$$

where $\lambda$ denotes the Lebesgue measure (notice that in the above sum only one term is non-zero). The sequence of $\mu_{\beta, n}$ is measurable with respect to the $\sigma$-field $\tilde{F}_{s}$ generated by the random variables $W_{J}, J \in \mathscr{D}_{s}$. 
For $\beta>0$, the corresponding partition functions $Z_{n}(\beta)$ (which are random variables) are given by

$$
Z_{n}(\beta)=c^{-n \beta} \sum_{I \in \mathscr{\mathscr { O }}_{n}} \prod_{\mathrm{s}=1}^{n} \sum_{J \in \mathscr{\mathscr { O }}_{\mathrm{s}}} W_{J}^{\beta} \lambda(I \cap J) c^{n} .
$$

The finite volume pressure $P_{n}(\beta)$ is defined as usual by

$$
P_{n}(\beta)=n^{-1} \log Z_{n}(\beta) .
$$

Using the analogy with the statistical mechanics we also define the finite volume random states $v_{\beta, n}$ to be the probability measures on the $\sigma$-field generated by $\mathscr{D}$ :

$$
v_{\beta, n}(\cdot)=Z_{n}(\beta)^{-1} \mu_{\beta, n}(\cdot) .
$$

We are interested in the thermodynamic (infinite volume) limit $n \rightarrow \infty$ of the above quantities.

In relation with the setting of the classical statistical mechanics, we can express the definition of the random measure $\mu_{\beta, n}(I)$ in terms of the random kernels $N_{n}(x, A)$ defined for every point $x \in[0,1]$ and every Borel set $A$ by

$$
N_{n}(x, A)=W_{I}^{\beta} \mathbb{1}_{I \cap A}(x) ; \quad I \in \mathscr{D}_{n}
$$

and such that

i) $x \mapsto N_{x}(x, A)$ is a Borel function for every $A$;

ii) $A \mapsto N_{x}(x, A)$ is countably additive $\forall x \in[0,1]$.

Using this definition, the corresponding measure $\mu_{\beta, n}(A)$ is given by

$$
\mu_{\beta, n}(A)=\int_{A} d x N_{1}(x, A) \cdots N_{n}(x, A) .
$$

It is worth noticing that contrary to the discrete potential theory these kernels are random and they depend on $n$.

The measure $\mu_{1, n}$ for the special case $\beta=1$ was first studied by Kahane and Peyrière [10] as an example of the Multiplicative Chaos. More precisely, they showed that under some weaker assumptions on the random variable $W$ the sequence of measures $\mu_{1, n}$ converges a.s. in the weak topology to a random measure $\mu$. Moreover, the measure $\mu$ is concentrated on Borel sets having Hausdorff dimension given by $1-E\left(W \log _{c} W\right)$ (where $E(\cdot)$ means the expectation) and the support of $\mu$ is the whole interval $[0,1]$. The random variables

$$
Z_{1, n}=\sum_{I \in \mathscr{Q}_{n}} \mu_{1, n}(I)
$$

define a positive (i.e. $\geqq 0$ ) integrable martingale which converges a.s. to the random limit $Z_{1, \infty}$ with $E\left(Z_{1, \infty}\right) \leqq 1$.

A generalisation of this result was given in [11], and can be rephrased as follows. For any integer $q$, we define an operator $K_{q}$ on the set of bounded positive measures $\mathscr{M}$ of $[0,1]$ by

$$
\left(K_{q} \lambda\right)(A)=\sum_{I \in \mathscr{D}_{q}} W_{I} \lambda(I \cap A) .
$$


It is easy to verify that on the $\sigma$-field generated by $\mathscr{D}_{p}$, we have

$$
\mu_{1, p}=\mathscr{K}_{p} \lambda
$$

where $\mathscr{K}_{p}=K_{p} \cdots K_{1} \cdot \mathscr{K}_{p}$, is an operator which maps the set $\mathscr{M}$ of bounded positive measures into random measures. It was shown in [11] that the random variables $\mathscr{K}_{p} \lambda$ converge weakly a.s. to the random measure

$$
\mu=\mathscr{K} \lambda=\lim _{p \rightarrow \infty} \mathscr{K}_{p} \lambda .
$$

The total mass of this limit measure is given by the random variable $Z_{1, \infty}$. In general, the limit $\mu$ is not absolutely continuous w.r.t. the measure $\lambda$. By zero-one law, the probability for this is either zero or one. If $\mathscr{K} \lambda=0$ a.s., we say that $\mathscr{K}$ is degenerate on $\lambda$. On the other hand, if the martingale $\mathscr{K}_{p} \lambda$ converges in $L^{1}(\Omega)$, we say that $\mathscr{K}$ is fully acting on $\lambda$; we define $\mathscr{K}$ as the Multiplicative Chaos operator. This is summarised in the following [11].

Theorem 2.1. Given $\mathscr{K}_{p}$ as above, there exists a Borel set $A$ such that

$$
E\left(\mu(A) \mid \mathscr{D}_{p}\right)=\mathbb{1}_{A} \mathscr{K}_{p} \lambda
$$

and $\lambda$ can be decomposed uniquely into a sum of two mutually singular measures $\lambda=\lambda_{1}+\lambda_{2}$ such that $\mathscr{K}$ acts fully on $\hat{\lambda}_{1}$ and is degenerated on $\lambda_{2}$. The operator E $\mathscr{K}$ mapping $\lambda$ into $\lambda_{1}$ is a projection.

The above result remains unchanged if instead of the Lebesgue measure $\lambda$ we consider any positive Radon measure.

These very remarkable results are reminiscent of statistical mechanics ones. In particular, one would like to interpret the measure $\mu$ as a Gibbs state at inverse temperature one. One of the differences is that $\mu$ is not a probability measure. Note however, that it is normalised in the average.

On the other hand, the thermodynamic formalism used for the study of local singularities of a measure in [2] has similarities with our problem. Namely, if we define the partition function for a measure $\sigma$ by

$$
\Psi_{n}(\beta)=\sum_{I \in \mathscr{D}_{n}} \sigma(I)^{\beta}
$$

it is known [5] that if the free energy $G(\beta)$ defined by

$$
\beta G(\beta)=\lim _{n \rightarrow \infty}-n^{-1} \log \Psi_{n}(\beta)
$$

exists and is $C^{1}$, the function $f(\alpha)$ is given by the Legendre transform of $G(\beta)$. Some interesting situations can occur if $G$ is not everywhere $C^{1}$. Roughly speaking, they correspond to phase transitions.

If we wish to use the thermodynamic formalism in the case of multiplicative chaos, the natural generalisation should be to define a measure $\tilde{\mu}_{\beta, n}(I)$ by

$$
\tilde{\mu}_{\beta, n}(I)=\mu_{1, n}(I)^{\beta}
$$

and the corresponding partition function by $\tilde{Z}_{n}(\beta)=\sum_{I \in \mathscr{Q}_{n}} \tilde{\mu}_{\beta, n}(I)$.

Notice however, that $\tilde{Z}_{n}(\beta)$ differs from the partition function $Z_{n}(\beta)$ of our model by the multiplicative constant $c^{n(1-\beta)}$. We shall, from now on, assume that 
$\forall p>1, E\left(W^{p}\right)<c^{p-1}$. It follows from [10] that the random variable $Z_{1, \infty}$ has moments of all orders.

It is worth noticing that the correct characterisation of the statistical mechanics is given with a measure defined by an appropriate limiting procedure. We define the multimeasure $\tilde{\mu}_{\beta, \infty}$ as the weak a.s. limit of the sequence of the random variables $\tilde{\mu}_{\beta, p}$ for $p \rightarrow \infty$. The partition function is given by

$$
Z_{n}^{*}(\beta)=\sum_{I \in \mathscr{D}_{n}} \tilde{\mu}_{\beta, \infty}(I)
$$

We will nevertheless use the partition function $\tilde{Z}_{n}(\beta)$ (or $\left.Z_{n}(\beta)\right)$ because from the next result it follows that the two partitions $Z_{n}^{*}(\beta)$ and $\tilde{Z}_{n}(\beta)$ give rise to the same pressure.

Proposition 2.2. If one of the limits $\lim _{n \rightarrow \infty} n^{-1} \log _{c} Z_{n}^{*}(\beta)$ or $\lim _{n \rightarrow \infty} n^{-1} \log _{c} \tilde{Z}_{n}(\beta)$ exists almost surely or in the mean, then the other one also exists and is equal to it.

Before proving the proposition let us remark that for $I \in \mathscr{D}_{n}$, the limit $\tilde{\mu}_{\beta, \infty}(I)$ can be decomposed as

$$
\tilde{\mu}_{\beta, \infty}(I)=\tilde{\mu}_{\beta, n}(I) \tilde{Y}_{I, \beta, \infty},
$$

where the random variable $\tilde{Y}_{I, \beta, \infty}=\left(Y_{I, 1, \infty}\right)^{\beta}$, measurable with respect to the tail $\sigma$-field, equals $\left(\lim _{p \rightarrow \infty} \sum_{J \in \mathscr{D}_{n+p}} \mu_{1, p}(J \cap I)\right)^{\beta}$. As we will see later, this decomposition of the limit measure yields to a natural interpretation of the variables $Y_{I, 1, \infty}$ as boundary conditions. Moreover, the advantage of this reformulation is that the terms $\tilde{Y}_{I, \beta, \infty}$ have a trivial dependence on the temperature.

The study of the variable $Y_{I, 1, \infty}$ is also encountered in some problems related to infinite particle systems. In particular, Durett and Liggett [4] studied in detail a simplified version of the smoothing process called smoothing transformation. This transformation is defined as follows. Let $W_{1}, \ldots, W_{k}$ be $k$ fixed non-negative random variables with Prob $\left(W_{i}>0\right)>0$ and arbitrary joint distributions, and $\mathscr{M}$ the class of all probability measures on $[0, \infty)$. If $X_{1}, \ldots, X_{k}$ are independent random variables with common distribution $v$, and independent of $\left(W_{1}, \ldots, W_{k}\right)$, the smoothing transformation $S$ on $\mathscr{M}$ is defined by letting $S v$ be the distribution of $W_{1} X_{1}+\cdots+W_{k} X_{k}$. Furthermore, $S$ can be viewed as a nonlinear transformation on the class of Laplace transforms of the elements of $\mathscr{M}$.

To prove the proposition we need the following

Lemma 2.3. $\forall q>0$, the random variable $1 / Y_{I, 1, \infty}^{q}$ belongs to $L^{1}$.

Proof. If we define the non-increasing function

$$
\phi(t)=E \exp \left(-t Y_{I, 1, \infty}\right)
$$

we have that

$$
E\left(1 / Y_{I, 1, \infty}^{q}\right) \leqq C_{q} \int_{1}^{\infty} \phi(t) \frac{d t}{t^{1-q}}
$$

Assuming now for a positive $\rho$, that $W>\rho$ a.s. Using the recursion relation $\phi(t)=E \phi(t W / c)^{c}$, it is easy to prove that there exists a constant $r>0$ such that $\phi(t) \leqq \exp \left(-r t^{\rho}\right) \forall t \geqq 1$ and therefore

$$
\int_{1}^{\infty} \phi(t) \frac{d t}{t^{1-q}}<\infty
$$


Proof of the Proposition 2.2. Using the decomposition of the limit for $\bar{\mu}_{\beta, \infty}(I)$, the partition function $Z_{n}^{*}(\beta)$ can be written as

$$
Z_{n}^{*}(\beta)=\sum_{I \in \mathscr{D}_{n}} \tilde{\mu}_{\beta, n}(I) \tilde{Y}_{I, \beta, \infty} .
$$

If $\forall \varepsilon>0$ and $\forall n \in N$, we consider the events (measurable with respect to $\mathscr{F}_{\infty}$ )

$$
A_{n, \varepsilon}=\bigcup_{I \in \mathscr{D}_{n}}\left\{\tilde{Y}_{I, 1, \infty}>e^{n \varepsilon}\right\} \quad \text { and } \quad E_{n, \varepsilon}=\bigcup_{I \in \mathscr{D}_{n}}\left\{\tilde{Y}_{I, 1, \infty}<e^{-n \varepsilon}\right\},
$$

from Chebyčev's inequality we have for $0<p<\infty$,

$$
\operatorname{Prob}\left(A_{n, \varepsilon}\right) \leqq \sum_{I \in \mathscr{O}_{n}} E\left(\tilde{Y}_{I, 1, \infty}^{p}\right) e^{-n \varepsilon p} \quad \text { and } \operatorname{Prob}\left(E_{n, \varepsilon}\right) \leqq \sum_{I \in \mathscr{D}_{n}} E\left(\tilde{Y}_{I, 1, \infty}^{-p}\right) e^{-n \varepsilon p} .
$$

We shall first prove the inequality

$$
\underset{n}{\limsup } n^{-1} \log _{c} Z_{n}^{*}(\beta) \leqq \underset{n}{\limsup } n^{-1} \log _{c} \tilde{Z}_{n}(\beta) \quad \text { a.s. . }
$$

Noting that we will have the inequalities

$$
\exp (-n \varepsilon \beta) \tilde{Z}_{n}(\beta) \leqq Z_{n}^{*}(\beta) \leqq \exp (n \varepsilon \beta) \tilde{Z}_{n}(\beta)
$$

once we show that Prob $\left(\left(\lim \sup A_{n, \varepsilon}\right)^{c} \cap\left(\lim \sup E_{n, \varepsilon}\right)^{c}\right)=1$. To get this assertion, let us first observe that from the preceding Lemma 2.3 and Theorem 2 of [10] we have the existence of all moments of the random variable $\tilde{Y}_{I, 1, \infty}$. Now, provided that $\log c<\varepsilon p$ and applying the Borel-Cantelli lemma the assertion follows. To complete the proof let us remark that the equalities for the expectations are a consequence of Fatou's lemma.

\section{High Temperature Behaviour}

In the following we study the high temperature region of the model introduced in the previous section. We define an inverse critical temperature $\beta_{c}$, and we prove that for $\beta<\beta_{c}$ (high temperature), the free energy exists in the thermodynamic limit and has the so called self-averaging property. Moreover, we prove the unicity of the random state $v_{\beta}$, and calculate its Hausdorff dimension.

For $\beta_{c}$ given by

$$
\frac{\beta_{c}}{E\left(W^{\beta_{c}}\right)} E\left(W^{\beta_{c}} \log _{c} W\right)=1+\log _{c} E\left(W^{\beta_{c}}\right)
$$

the high temperature region is defined by $\beta<\beta_{c}$.

We begin by defining the sequence of random variables

$$
X_{n}(\beta)=\frac{Z_{n}^{*}(\beta)}{E Z_{n}^{*}(\beta)} .
$$

One can easily see that for $\beta=1$, the above variables define a non-negative $\mathscr{F}_{n}$-martingale satisfying $E X_{n}(1)=1$. Moreover, $X_{n}(1)$ converges a.s. to the random variable $X_{\infty}$ satisfying $E X_{\infty}=1$ and one can prove by a standard argument on the tail $\sigma$-field that $\operatorname{Prob}\left(X_{\infty}=0\right)=0$. 
Let us'moreover consider the following sequence of random variables which is easily seen to define a $\mathscr{F}_{n}$-martingale

$$
V_{n}(\beta)=c^{-n} \sum_{I \in \mathscr{D}_{n}} \prod_{s=1}^{n} \sum_{J \in \mathscr{D}_{s}} \frac{W_{J}^{\beta}}{E\left(W_{J}^{\beta}\right)} \lambda(I \cap J) c^{n} .
$$

Before presenting our results we recall that an integrable martingale converging in $L^{1}(\Omega)$, is called regular [16].

Lemma 3.1. For $0<\beta<\beta_{c}$, the martingale $V_{n}(\beta)$ defined above is regular. Moreover, for every $n, V_{n}(\beta)=E\left(V_{\infty}(\beta) \mid \mathscr{F}_{n}\right)$ a.s.

Proof. According to Doob's martingale convergence theorem [3], and since $E\left(V_{n}(\beta)\right)=1$, the martingale $V_{n}(\beta)$ converges a.s. to an integrable random variable $V_{\infty}(\beta)$. We then only need to prove that $V_{n}(\beta)$ is a uniformly integrable martingale. Using Theorem 1 of [10], it is enough to prove that $E V_{\infty}(\beta)>0$. The idea is to consider the supermartingale $V_{n}^{1 / 2}(\beta)$ for which the bound

$$
(c-1)\left(E V_{n-1}^{1 / 2}(\beta)\right)^{2} \geqq 1-E\left(\frac{W^{\beta}}{E\left(W^{\beta}\right)} \log _{c} \frac{W^{\beta}}{E\left(W^{\beta}\right)}\right)
$$

can be deduced from the proof of the theorem 1 in $[10]$. Since $E V_{n}(\beta)=1$ by definition, the variables $V_{n}^{1 / 2}(\beta)$ are uniformly integrable i.e.

$$
\lim _{n} E V_{n}^{1 / 2}(\beta)=E V_{\infty}^{1 / 2}(\beta) \neq 0 \text {. }
$$

As a consequence of the previous lemma and the definition of the critical temperature $\beta_{c}$ we obtain the

Corollary 3.2. For $\beta<\beta_{c}$, the sequence of random variables $X_{n}(\beta)$ converges almost surely and in $L^{1}$ to a positive integrable random variable $X_{\infty}(\beta)$.

Proof. We first note that $E \tilde{Y}_{I, \beta, \infty}<\infty$. Moreover, for every $n, E\left(\tilde{Y}_{I, \beta, \infty} \mid \tilde{F}_{n-1}\right)$ $=E \tilde{Y}_{I, \beta, \infty}$ and this implies

$$
E\left(X_{n}(\beta) \mid \mathscr{F}_{n-1}\right)=E\left(V_{n}(\beta) \mid \mathscr{F}_{n-1}\right)=V_{n-1},
$$

proving that the martingale $V_{n}(\beta)$ is a modification of the sequence of random variables $X_{n}(\beta)$ and hence the result follows.

We have the

Theorem 3.3. For $\beta<\beta_{c}$, the limit $\lim _{n \rightarrow \infty} n^{-1} \log _{c} X_{n}(\beta)$ exists almost surely and in the mean and it is equal to zero.

Proof. From the previous corollary and using Jensen's inequality, we have

$$
E\left(\log V_{n}(\beta)\right)-\log E\left(\tilde{Y}_{I, 1, \infty}\right)+E\left(\log \tilde{Y}_{I, 1, \infty}\right) \leqq E\left(\log X_{n}(\beta)\right) \leqq 0 .
$$

The assertion now is proved using Lemma 2.3 and Corollary 3.2 and remarking that $0 \geqq E\left(\log V_{n}(\beta)\right) \geqq E\left(\log V_{\infty}\right)>-\infty$.

Lemma 3.4. For $\beta<\beta_{\text {c }}$ the limit

$$
\lim _{n \rightarrow \infty} n^{-1} \log _{c} E \tilde{Z}_{n}(\beta)
$$


exists and defines an analytic and strictly convex function of $\beta$ given by $\log _{c} E\left(W^{\beta}\right)-\beta+1$.

Proof. From the definition of the partition function $\tilde{Z}_{n}(\beta)$, one can easily see that $E\left(\tilde{Z}_{n}(\beta)\right)$ is equal to $c^{\beta(n-1)} E\left(W^{\beta}\right)^{n}$. Now, using Hölder's inequality we have that $\log _{c} E\left(W^{\beta}\right)$ is a strictly convex function on $\mathbf{R}$. On the set $\left\{\beta: \log _{c} E\left(W^{\beta}\right)<\infty\right\}$, the Lebesgue dominated convergence theorem implies the analyticity of $\log _{c} E\left(W^{\beta}\right)$.

For the pressure we can now prove the

Theorem 3.5. For $0<\beta<\beta_{c}$, the limit

$$
P(\beta)=\lim _{n \rightarrow \infty} n^{-1} \log _{c} Z_{n}^{*}(\beta)
$$

exists almost surely. Moreover, $P(\beta)=\log _{c} E\left(W^{\beta}\right)-\beta+1$.

Proof. The a.s. convergence of the random variable $X_{n}(\beta)$ to a nonzero limit, guarantees the a.s. positivity of $X_{\infty}(\beta)$ and therefore the a.s. existence of $\log _{c} X_{\infty}(\beta)$. Hence, $\lim _{n \rightarrow \infty} n^{-1} \log _{c} X_{n}(\beta)=0$ and the previous lemma, imply the existence of the pressure and its equality to $\log _{c} E\left(W^{\beta}\right)-\beta+1$.

In the physics terminology, the above result shows the self-averaging property of the free energy at high temperature (i.e. the quenched

and the annealed

$$
\lim _{n \rightarrow \infty} n^{-1} E \log _{c} Z_{n}^{*}(\beta)
$$

$$
\lim _{n \rightarrow \infty} n^{-1} \log _{c} E Z_{n}^{*}(\beta)
$$

limits agree):

Proposition 3.6. For $0<\beta<\beta_{c}$, the free energy given by $-\beta F(\beta)=P(\beta)$, is almost surely equal to the annealed mean.

We can now discuss the above result in terms of boundary conditions. One can define as boundary condition any family of random measures $b_{n}$ whose restrictions to each interval $I$ on the $n$-order partition is given in a similar way as for the measure $\tilde{\mu}_{\beta, n}$. The variables $\tilde{Y}_{I, \beta, \infty}$ and $c^{n} b_{n}(I)$ are mutually independent and have the same distribution. From this definition, two other random measures can be considered as typical boundary conditions; the fixed point $\tilde{Y}_{I, 1, \infty}$ of the smoothing transformation defined in Sect. 2 and the constant 1. For these boundary conditions, we have the

Theorem 3.7. For every temperature $\beta$, the free energy $F(\beta)$ is independent of the typical boundary conditions defined as above.

We cannot claim, for the moment, that the previous theorem holds in the case of more general boundary conditions. However, we conjecture that this is the case.

We can now investigate the thermodynamic limit of the random states defined in Sect. 2. We have the following

Theorem 3.8. For $0<\beta<\beta_{c}$, the sequence of random probability measures $v_{\beta, n}(\cdot)$ $=Z_{n}^{*}(\beta)^{-1} \tilde{\mu}_{\beta, \infty}(\cdot)$ converges almost surely to a random probability measure $v_{\beta}$ on the Borel field of $[0,1]$, as $n \rightarrow \infty$. 
Proof. It suffices to study $v_{\beta, n}$ over an arbitrary $c$-adic interval $J$ of the $k^{\text {th }}$ generation with $n>k$. Now, defining $X_{\beta, n}(J)=c^{n} E\left(\tilde{\mu}_{\beta, \infty}\right)^{-1} \tilde{\mu}_{\beta, \infty}(J)$ the random probability of $J$ is given by

$$
v_{\beta, n}(J)=\frac{X_{\beta, n}(J)}{X_{n}(\beta)} .
$$

Both the numerator and the denominator of the r.h.s. converge a.s. Moreover, by Corollary 3.2 we have that $X_{\infty}(\beta)>0$ if $\beta<\beta_{c}$.

One can see that the probabilities $v_{\beta}$ are projective limits verifying the compatibility conditions. Kolmogorov's extension theorem implies the existence of a unique limit on the Borel field of $[0,1]$.

In this way, we have established the analogue of the Gibbs measures; one can see the limits $v_{\beta}$ as the Gibbs measures associated to the Lebesgue measure. On the other hand, in the language of statistical mechanics the above results are independent of the boundary conditions. It is however not known if we can formulate DLR equations for the present model. Indeed, here we have studied laws on finite partitions instead of local specifications considered in the DLR formulation. This is rather natural for the version of the model we use: one can see our model as a long range interaction one. Hence, our problem is more intrinsic than the DLR one, where we have a liberty in the choice of boundary conditions. However, as we shall see, even without this liberty we have a phase transition.

In Multiplicative Chaos language, for $0<\beta<\beta_{c}$, the operator $\mathscr{K}$ acts fully on the Lebesgue measure of the interval $I$. Or, in an equivalent way, the measure $v_{\beta}$ is $\mathscr{K}$-regular. This regularity condition [10] allows to calculate the Hausdorff dimension $D_{H}$ of $v_{\beta}$ :

$$
D_{H}(\beta)=1-\beta \frac{1}{E\left(W^{\beta}\right)} E\left(W^{\beta} \log _{c} W\right)+\log _{c} E\left(W^{\beta}\right) .
$$

Note however, that the support of $v_{\beta}$ is the whole interval $[0,1]$.

The limit of $Z_{n}^{*}(\beta)$ can also be regarded as a singular (with respect to the Lebesgue) measure for $\beta \neq 0$. A natural question related to the behaviour of this singularity is whether the partition function becomes more or less singular as the temperature grows. Another question, relevant for the random states, is about the manner the partition function spreads its mass over its support (as a function of temperature). As we shall see in the following section, the partition function is singular at the critical temperature. On the other hand, one can see the definition of the critical temperature given at the beginning of this section as a condition involving entropy. This relation between the singularity of a measure and the entropy is a rather general phenomenon encountered in the study of the asymptotic behaviour of the measure valued processes. In a subsequent work we generalise the Multiplicative Chaos to a measure valued process and discuss this relation [14].

\section{Low Temperature Behaviour}

In this section we give a description of the low temperature $\left(\beta \geqq \beta_{c}\right)$ behaviour of the model. We start by proving that the pressure exists, in the thermodynamic limit and give an explicit formula. 
Lemma 4.1. For $\beta \geqq \beta_{c}$, the martingale $V_{n}(\beta)$ defined in the previous section converges a.s. to zero for $n \rightarrow \infty$.

Proof. We have that for $\beta \geqq \beta_{c}$,

$$
E\left(\frac{W^{\beta}}{E\left(W^{\beta}\right)} \log \frac{W^{\beta}}{E\left(W^{\beta}\right)}\right) \geqq \log c .
$$

It follows from Theorem 1 in [10] that $E V_{\infty}=0$, therefore the positive martingale $V_{n}(\beta)$ cannot be regular.

From this we have the

Corollary 4.2. For $\beta \geqq \beta_{c}$ the sequence of random variables $X_{n}(\beta)$ converges a.s. to a degenerate limit $X_{\infty}(\beta)$ and we have that $\operatorname{Prob}\left(X_{\infty}=0\right)=1$.

In order to calculate the pressure, we need some precise estimates on the rate at which the partition function $Z_{n}^{*}(\beta)$ is tending to 0 . To do this, we express the partition function as follows. Consider a locally finite rooted tree $\mathscr{T}$ with $c$ branches at each vertex, and associate at each edge the random "weight" $\tilde{W} \equiv W^{\beta} / c^{\beta}$. The total weight $S_{b}(\beta)$ for a branch $b$ of length $|b|=n$, is given by the roduct of the weights over the $n$ edges. The partition function can now be written as

$$
Z_{n}^{*}(\beta)=\sum_{b \in \mathscr{T},|b|=n} S_{b}(\beta) .
$$

With the preceding in mind, we will ask about the behaviour of the variables $S_{b}(\beta)$ for $\beta>\beta_{c}$. In particular, we will use Chernoff-type estimates for the probabilities of the large deviations of $S_{b}(\beta)$.

Let $P_{n}^{*}(\beta)$ be the sequence of random functions defined by

$$
P_{n}^{*}(\beta)=n^{-1} \log Z_{n}^{*}(\beta) .
$$

We only consider $\beta>0$ although some of the results are also valid for $\beta<0$. We have the following

Lemma 4.3. Uniformly in $n$ and in the randomness, the functions $P_{n}^{*}(\beta)$ are Lipschitz in $\beta$.

Proof. Let $\beta_{1}$ and $\beta_{2}$ be two positive numbers. From our hypothesis, it is easy to verify that there is a number $a \in] 0,1[$ such that for $b \in \mathscr{T},|b|=n$,

$$
a^{n\left|\beta_{2}-\beta_{1}\right|} S_{b}\left(\beta_{2}\right) \leqq S_{b}\left(\beta_{1}\right) \leqq S_{b}\left(\beta_{2}\right) a^{-n\left|\beta_{2}-\beta_{1}\right|} \text {. }
$$

Therefore, for any realisation of the randomness we have

$$
a^{n\left|\beta_{2}-\beta_{1}\right|} Z_{n}^{*}\left(\beta_{2}\right) \leqq Z_{n}^{*}\left(\beta_{1}\right) \leqq Z_{n}^{*}\left(\beta_{2}\right) a^{-n\left|\beta_{2}-\beta_{1}\right|}
$$

and the result follows.

As a consequence we have the following two corollaries.

Corollary 4.4. The functions $\operatorname{EP}_{n}^{*}(\beta)$ are uniformly Lipschitz.

Corollary 4.5. For a fixed value of the randomness, any accumulation point of the sequence $P_{n}^{*}(\beta)$ is a Lipschitz and convex function of $\beta$. The same is true for any accumulation point of the sequence $E P_{n}^{*}(\beta)$. 
Proof. We first have the trivial uniform bound

$$
\beta \log a \leqq P_{n}^{*}(\beta) \leqq-\beta \log a
$$

The existence of finite and Lipschitz accumulation points follow from the AscoliArzelà theorem, and the convexity follows from the existence of the limit on subsequences. A similar argument can be applied to the sequence $E P_{n}^{*}(\beta)$.

Remark. At this point it is not even clear that the accumulation points can be extended to measurable functions of the randomness. This property will be a consequence of our final result.

Let $P^{*}(\beta)$ be one of the previous accumulation points with fixed randomness. We are going to prove now a general result on its behaviour as a function of $\beta$. This result will be true almost surely with respect to the randomness.

Proposition 4.6. Let $\beta>\beta_{1}>0$. Almost surely, for any accumulation point of $P_{n}^{*}(\beta)$ (with fixed randomness), we have

$$
P^{*}(\beta) \leqq P^{*}\left(\beta_{1}\right)+\left(\frac{\beta}{\beta_{1}}-1\right) \log E\left(W^{\beta_{1}}\right)+\left(\frac{\beta}{\beta_{1}}+\beta_{1}-\beta-1\right) \log c
$$

Proof. Let for $b \in \mathscr{T}$ and $|b|=n$,

$$
\bar{S}_{b}(\beta)=\frac{S_{b}(\beta) c^{-n \beta / \beta_{1}}}{E S_{b}\left(\beta_{1}\right) c^{\beta / \beta_{1}}}=\frac{S_{b}\left(\beta_{1}\right)^{\beta / \beta_{1}} c^{-n \beta / \beta_{1}}}{E S_{b}\left(\beta_{1}\right)^{\beta / \beta_{1}}} .
$$

Let $\alpha=\beta_{1} / \beta<1$. From the definition of $S_{b}$, we have immediately

$$
E\left(\overline{S_{b}}(\beta)\right)^{\alpha}=\frac{1}{c^{n}} .
$$

It follows from Chebyčev's inequality that

$$
\operatorname{Prob}\left(\overline{S_{b}}(\beta)>n^{2 / \alpha}\right)=\operatorname{Prob}\left(\overline{S_{b}}(\beta)^{\alpha}>n^{2}\right) \leqq \frac{1}{n^{2} c^{n}} .
$$

For the measurable set $A_{n}$ defined by

$$
A_{n}=\left\{b \in \mathscr{T}:|b|=n, \overline{S_{b}}(\beta) \leqq n^{2 / \alpha}\right\},
$$

we have

$$
\operatorname{Prob}\left(A_{n}\right) \geqq 1-\frac{1}{n^{2}}
$$

Then, we have on $A_{n}$

$$
\bar{S}_{b}(\beta)=\bar{S}_{b}(\beta)^{\alpha} \overline{S_{b}}(\beta)^{1-\alpha} \leqq n^{\frac{2(1-\alpha)}{\alpha}} \overline{S_{b}}(\beta)^{\alpha}=n^{\frac{2(1-\alpha)}{\alpha}} \overline{S_{b}}\left(\beta_{1}\right)
$$

Therefore,

$$
Z_{n}^{*}(\beta) \leqq Z_{n}^{*}\left(\beta_{1}\right) n^{\frac{2(1-\alpha)}{\alpha}} E\left(W^{\beta_{1}}\right)^{n\left(\beta / \beta_{1}-1\right)} c^{n\left(\beta / \beta_{1}+\beta_{1}-\beta-1\right)},
$$

and remarking that $\operatorname{Prob}\left(\liminf A_{n}\right)=1$ by Borel Cantelli lemma, the result follows. 
Corollary 4.7. The same inequality is true for any accumulation point of $\operatorname{EP}_{n}^{*}(\beta)$.

Proof. This follows immediately from the above result since the functions $P_{n}^{*}(\beta)$ are uniformly bounded above and below.

We now apply this result for $\beta_{1}=\beta_{c}$.

Theorem 4.8. Almost surely for $\beta>\beta_{c}$,

$$
P^{*}(\beta)=\frac{\beta}{\beta_{c}} \log E\left(W^{\beta_{c}}\right)+\beta\left(\frac{1}{\beta_{c}}-1\right) \log c .
$$

Proof. Consider an accumulation point $P^{*}(\beta)$ of a sequence $P_{n}^{*}(\beta)$ with fixed randomness chosen in the set $\lim \inf A_{n}$, where $A_{n}$ is the sequence defined in Proposition 4.6. From Lemma $4.3 P^{*}(\beta)$ is a Lipschitz and convex function. Moreover, if $\beta \leqq \beta_{c}$, we can assume that

$$
P^{*}(\beta)=P_{T}(\beta),
$$

where $P_{T}(\beta)=\log E\left(W^{\beta}\right)+(1-\beta) \log c$. The function $P_{T}(\beta)$ is convex and differentiable for $\beta>\beta_{c}$. Since $P^{*}(\beta)$ is also convex, its graph must not be below the tangent to $P_{T}$ at $\beta=\beta_{c}$. Therefore we must have

$$
P^{*}(\beta) \geqq P_{T}\left(\beta_{c}\right)+\left(\beta-\beta_{c}\right) P_{T}^{\prime}\left(\beta_{c}\right) .
$$

From the definition of $\beta_{c}$, we have

$$
P_{T}^{\prime}\left(\beta_{c}\right)=\frac{E\left(W^{\beta_{c}} \log W\right)}{E\left(W^{\beta_{c}}\right)}-\log c=\frac{1}{\beta_{c}} \log E\left(W^{\beta_{c}}\right)+\left(\frac{1}{\beta}-1\right) \log c
$$

which implies

$$
\begin{aligned}
P^{*}(\beta) \geqq & \log E\left(W^{\beta_{c}}\right)+\left(1-\beta_{c}\right) \log c \\
& +\left(\beta-\beta_{c}\right)\left(\frac{1}{\beta_{c}} \log E\left(W^{\beta_{c}}\right)+\left(\frac{1}{\beta_{c}}-1\right) \log c\right) \\
= & \frac{\beta}{\beta_{c}} \log E\left(W^{\beta_{c}}\right)+\left(\frac{\beta}{\beta_{c}}-\beta\right) \log c .
\end{aligned}
$$

On the other hand, using Proposition 4.6 we have

$$
\begin{gathered}
P^{*}(\beta) \leqq \\
+\log E\left(W^{\beta_{c}}\right)+\left(1-\beta_{c}\right) \log c+\left(\frac{\beta}{\beta_{c}}-1\right) \log E\left(W^{\beta_{c}}\right) \\
\left.+\beta_{c}-\beta-1\right) \log c \\
=\frac{\beta}{\beta_{c}} \log E\left(W^{\beta_{c}}\right)+\left(\frac{\beta}{\beta_{c}}-\beta\right) \log c,
\end{gathered}
$$

and the result follows.

We can now estimate the pressure. 
Corollary 4.9. For $\beta>\beta_{c}$,

$$
n^{-1} \log _{c} Z_{n}^{*}(\beta) \underset{n \rightarrow \infty}{\longrightarrow} \frac{\beta}{\beta_{c}} \log _{c} E\left(W^{\beta_{c}}\right)+\left(\frac{\beta}{\beta_{c}}-\beta\right)
$$

almost surely. The same result is valid for the expectation.

Proof. If follows from Theorem 4.8 that almost surely all the accumulation points are equal to the same constant.

For the free energy we have

Proposition 4.10. For $\beta>\beta_{c}$, the free energy $F(\beta)$ equals $-\left(1 / \beta_{c}\right) \log _{c} E\left(W^{\beta_{c}}\right)$ $-\left(1 / \beta_{c}\right)+1$ almost surely.

It should be mentioned at this point that one can interpret as order parameter the probability of the event $\left\{X_{\infty}(\beta)=0\right\}$ related to the limiting behaviour of the partition function. We have shown that for $\beta<\beta_{c}$, Prob $\left(X_{\infty}(\beta)=0\right)=0$ and at low temperature $\operatorname{Prob}\left(X_{\infty}(\beta)=0\right)=1$. So, one can characterise the phase transition of our model by this order parameter, but our study of the critical behaviour is more complete. This remark is natural if one wishes to relate our model to critical branching processes, since the total mass of the measure is changed.

We have computed the free energy of the system for all positive temperatures. We can now draw some conclusions on the dimension spectrum of the measure. Since the free energy is $C^{1}$, we conclude that every singularity exponent $\alpha$ belonging to the interval $\left[\alpha_{c}, 1\right]$ occurs on a set of Hausdorff dimension $f(\alpha)$, where

$$
\alpha_{c}=\frac{1}{\beta_{c}}\left(\log c-\frac{E\left(W^{\beta_{c}} \log W\right)}{E\left(W^{\beta_{c}}\right)}\right)=\frac{1}{\beta_{c}}\left(\log c\left(1-\frac{1}{\beta_{c}}\right)-\frac{1}{\beta_{c}} \log E\left(W^{\beta_{c}}\right)\right),
$$

and $f(\alpha)$ is the Legendre transform of $F(\beta)$. Since the phase transition is not of first order $\left(F\right.$ is $\left.C^{1}\right)$, we also conclude that the singularities of order $\alpha<\alpha_{c}$ cannot occur at least on sets of positive Hausdorff dimension. Note also that $f\left(\alpha_{c}\right)=\alpha_{c} \beta_{c}$ $-F\left(\beta_{c}\right)=0$. See also $[10]$.

\section{References}

1. Aizenman, M., Lebowitz, J.L., Ruelle, D.: Some Rigorous Results on the SherringtonKirkpatrick Spin Glass Model. Commun. Math. Phys. 112, 3-20 (1987)

2. Collet, P., Lebowitz, J.L., Porzio, A.: The Dimension Spectrum of Some Dynamical Systems. J. Stat. Phys. 47, 609-644 (1987)

3. Dellacherie, C., Meyer, P.A.: Probabilités et potentiel. Paris: Hermann (1980)

4. Durret, R., Liggett, T.M.: Fixed Points of the Smoothing Transformation. Z. Wahr. Verw. Geb. 64, 275-300 (1983)

5. Ellis, R.S.: Large deviations for a general class of random vectors. Ann. Prob. 12, 1-12 (1984)

6. Frostman, O.: Potentiel d'équilibre et capacité des ensembles. Håkan Ohlsson, Lund 1935

7. Guivarc'h, Y.: Sur une extension de la loi semi-stable fonctionnelle non linéaire de Benoit Mandelbrot. Ann. Inst. Henri Poincaré 26, 261-285 (1990)

8. Hasley, T.C., Jensen, M.H., Kadanoff, L.P., Proccacia, I., Shraiman, B.I.: Fractal measures and their singularities: The characterization of strange sets. Phys. Rev. B33, 1141-1151 (1986)

9. Holley, R., Liggett, T.M.: Generalized potlatch and smoothing processes. Z. Wahr. Verw. Geb. 55, 165-195 (1981). 
10! Kahane, J.-P., Peyrière, J.: Sur certaines martingales de Benoit Mandelbrot. Adv. Math. 22, 131-145 (1976)

11. Kahane, J.-P.: Multiplications aléatoires et dimensions de Hausdorff. Ann. Inst. Henri Poincaré 23, 289-296 (1987)

12. Kahane, J.-P., Katznelson, Y.: Décomposition des mesures selon la dimension. Colloquium Mathematicum vol. LVIII, fasc. 2, 269-279 (1990)

13. Koukiou, F.: Rigorous bounds for the free energy of the short-range Ising spin glass model. Europhys. Lett. 17, 669-671 (1992)

14. Koukiou, F.: Random Interactions, Critical Behaviour and Measure Valued Processes (In preparation)

15. Mandelbrot, B.B.: In Statistical models and turbulence. Symposium at U.C. San Diego 1971, Berlin, Heidelberg, New York: Springer 1972

16. Neveu, J.: Discrete-parameter martingales. Amsterdam: North-Holland 1975

17. Perkins, E.A.: A space-time property of a class of measure-valued branching diffusions. Trans. Am. Math. Soc. 305, 743-795 (1988)

Communicated by M. Aizenman 